\title{
REFLEXÕES SOBRE O PROCESSO DE CUIDAR DA ENFERMAGEM EM SAÚDE MENTAL
}

\author{
REFLEXIONS ABOUT THE NURSING WORKING PROCESS \\ IN MENTAL HEALTH
}

Célia Maria Sivalli Campos* Sônia Barros**

CAMPOS, C.M.S.; BARROS, S. Reflexões sobre o.processo de cuidar da enfermagem em saúde mental.

Rev.Esc.Enf.USP, v.34, n.3, p. 271-6, set. 2000.

\section{RESUMO}

Trata-se de um ensaio sobre o processo de trabalho da enfermagem em saúde mental. Diversas concepções têm fundamentado a assistência psiquiátrica, assim como as propostas de reorientação dessas práticas; a enfermagem tem suas práticas referidas a esse processo. É a visão de mundo dos profissionais que determina a escolha de uma determinada tendência e o entendimento do processo saúde-doença. A necessidade de rever o seu objeto de trabalho, assim como sua prática frente às transformações na assistência em saúde mental, tem apontado para novas posturas profissionais frente ao sujeito com transtornos psíquicos, assim como para a aquisição de conhecimento para além dos adquiridos nos cursos de graduação.

PALAVRAS-CHAVE: Saúde Mental.Assistência de enfermagem.

\section{ABSTRACT}

This is an essay about the nursing working process in mental health. Several conceptions have substantiated the psychiatric assistance, as well as the proposals of reorientation of those practices; nursing practices have been related to that process. The professionals' standpoint determines the choice of a tendency and the understanding of the healthillness process. The need of reviewing the nursing working object, as well as its practice in view of the transformations in mental health assistance, has pointed towards new professional attitudes regarding the person with psychic disorders, as well as towards the acquisition of knowledge besides that one obtained at college courses.

KEYWORDS: Mental Health. Nursing assistance.

\section{1- Processo de cuidar da enfermagem em saúde mental: origem e situação atual}

Dada a complexidade que representa um indivíduo acometido por um transtorno psíquico severo - objeto das intervenções em saúde mental têm sido, cada vez mais, reconhecidas as vantagens da execução das práticas assistenciais em saúde mental por um conjunto de profissionais, a equipe. Entende-se o trabalho em equipe aquele realizado por um conjunto diversificado de profissionais de diferentes áreas e não o trabalho realizado individualmente por cada um deles.
Então, com base na "divisão do trabalho, a nenhum agente isolado cabe mais realizar a totalidade das ações necessárias ao conjunto da assistência. A interdependência $e$ a complementaridade, cada vez maior entre os vários trabalhos parcelares, permite a constituição de uma nova totalidade" (PEDUZZI; PALMA, 1996: 236).

O parcelamento do trabalho na assistência á saúde originou-se da incorporação tecnológica ao processo de produção. O trabalhador médico

* Enfermeira, doutoranda em enfermagem, professora assistente da Escola de Enfermagem-USP; professora assistente da Faculdade de Enfermagem da Universidade de Mogi das Cruzes Endereço eletrônico para contato: celiasiv@usp.br

** Enfermeira, doutora em enfermagem, professora doutora da Escola de Enfermagem-USP, representante da Escola de Enfermagem da USP no Projeto de Integração Docente Assistencial - PIDA, da USP com a Secretaria de Estado da Saúde, no Centro de Atenção Psicossocial (CAPS) Dr Luiz da Rocha Cerqueira.Endereço eletrônico para contato: sobarros@usp.br 
coletivo dividiu-se "horizontalmente", tanto em diferentes especialidades dentro da prática médica, quanto em agregação de outros profissionais com práticas complementares, como o psicólogo, o terapeuta ocupacional, o assistente social, o administrador, o odontólogo (GONÇALVES, 1992).

Assim, com a divisão pormenorizada do trabalho nas práticas de saúde, o trabalho da enfermeira surgiu como retaguarda para o trabalho do médico. Ele era composto principalmente de atividades manuais, associadas ao processo terapêutico (GONÇALVES, 1992).

Pode-se afirmar que, na organização das práticas de saúde, o modelo médico é hegemônico (DONNANGELO; PEREIRA, 1976; GONÇALVES, 1992, 1976; SPINK, 1992). Logo, as transformações das práticas de enfermagem psiquiátrica acompanharam as ocorridas na psiquiatria, enquanto prática do médico, porém acrescida de outros saberes, como os da psicologia, da sociologia, entre outros.

Atualmente, as práticas dirigidas à assistência em saúde mental estão referidas às várias tendências, concepções coexistentes e determinantes do entendimento acerca do processo saúde-doença e coerentemente a este, à organização das instituições que, por sua vez, determinam a natureza da assistência aos seus usuários (AMARANTE, 1992). A enfermagem, evidentemente, não está imune a esse processo e tem suas práticas referidas a ele.

E como está inserida a prática da enfermagem junto aos outros agentes do trabalho em saúde mental?

Pode-se afirmar que as práticas assistenciais à saúde mental têm passado por diferentes orientações ao longo do tempo.

As propostas de reorientação na assistência de enfermagem à saúde mental foram determinadas pelas mudanças ocorridas na psiquiatria, que segundo ROCHA (1994), foram:

\section{- Psiquiatria Organicista/Biológica}

Teve suas bases na "revolução Pineliana", do final do século XVIII, quando a internação passou a ter caráter médico. A psiquiatria passou a ser uma disciplina médica, a primeira especialidade médica, que passou a preocupar-se com a classificação das patologias. O hospital passou então a ser o espaço institucional definido para a observação dos sinais e sintomas da loucura. A psiquiatria organicista originou-se no hospital.

No final do século XIX Kraepelin deu uma classificação às doenças psiquiátricas, e empregou

metodologia semelhante à utilizada na medicina. A sede da doença era o corpo, que passou a ser o objeto da psiquiatria, e no qual eram feitas as intervenções terapêuticas. Eram elas: malarioterapia, banhos, sangrias, posteriormente a sonoterapia, insulinoterapia, lobotomia e eletroconvulsoterapia, evoluindo para os psicofármacos, que passaram a partir de 1952, até os nossos dias, a ser o instrumento mais utilizado na prática do médico.

Nesta concepção, o tratamento tem a finalidade de restaurar o indivíduo acometido a um padrão de normalidade previamente determinado, geralmente associado à redução dos sintomas que

o levaram à internação, utilizando para isso o diagnóstico e a terapêutica, ações geralmente prescritas pelo médico e executadas pelos outros profissionais da equipe, os quais são coadjuvantes

e ficam impossibilitados de apropriarem-se da unidade concepção-execução do tratamento, que fica em poder do profissional médico. Este é o modelo hegemônico nas práticas assistenciais psiquiátricas no Brasil até os dias de hoje.

\section{- Psiquiatria psicoterápica}

Influenciada pela psicanálise, que trouxe à cena a existência de um mundo mental, o inconsciente, a psiquiatria psicoterápica propunha o entendimento da doença mental como patologia do psiquismo, em contraposição à psiquiatria organicista.

A importância de compreender a pessoa, mesmo que acometida por um transtorno mental, enquanto situada no tecido das relações sociais sujeito de sua história - e não somente descrever suas doenças, foi trazida por Freud (1856-1939), que revolucionou o entendimento da loucura por meio da psicanálise. Passou a ser importante ouvir esses sujeitos, saber de suas histórias, interpretando os seus significados para além do discurso manifesto. Nessa concepção o louco passou a ser sujeito e dono da sua história.

\section{- Psiquiatria social}

Teve início em decorrência da necessidade de restabelecer prontamente a mão de obra que atuaria na reconstrução dos países no período pós II Guerra Mundial, combinada à constatação dos efeitos deletérios que exerciam os hospitais aos pacientes. A apatia, a perda da capacidade de trabalho e da autonomia, eram citadas como uma segunda doença, produzida nos hospitais (BANDEIRA, 1991). Fazia-se necessário trans- 
formar o espaço hospitalar, comparado com os campos de concentração, em local de tratamento.

Leis foram promulgadas, direcionadas à novas formas de assistência ao doente mental. Surgiu na Inglaterra o "National Health Service" e o "Disabled Persons Act", em 1944, e em 1945, na França, a Segurança Social. Com a nova organização de saúde, passou a ser absurda a idéia de desperdício da força de trabalho, representada pelo contingente de pessoas depositadas nos hospícios e asilos.

Foi um movimento que propôs-se a transformar o espaço hospitalar em espaço terapêutico. Esse movimento deu origem a:

\section{Comunidade Terapêutica:}

Protagonizada por Maxwell Jones, que propunha-se a capitalizar "conscientemente no tratamento os recursos da instituição, da equipe, dos pacientes e de seus parentes. Isso implica, portanto, acima de tudo uma mudança no status comum dos pacientes. Em colaboração com a equipe, tornam-se participantes ativos em sua própria terapia, na de outros pacientes e em muitos aspectos das atividades gerais da unidade" (JONES, 1972:89).

\section{Psiquiatria Preventiva:}

Passou a incluir, além dos "doentes", um grupo maior sob seus auspícios. Surgiu a saúde mental, articulada aos conceitos da saúde pública. O espaço de atuação da Psiquiatria Preventiva passou a ser a comunidade, e seu objeto de intervenção os indivíduos considerados susceptiveis ao desenvolvimento de um agravo mental. As intervenções foram divididas, por niveis de complexidade, em prevenção primária, secundária e terciária, numa transposição desses niveis de prevenção e dos conceitos de história natural da doença, para a saúde mental (CAPLAN, 1980).

\section{Psiquiatria de Setor:}

Foi pensada e implantada como movimento de contestação da psiquiatria asilar.

A idéia era levar a psiquiatria à população, possibilitando ao individuo acometido por um transtorno psiquiátrico um tratamento na sua comunidade. A internação em hospital psiquiátrico deveria ser vista como um evento transitório. $\mathrm{O}$ hospital psiquiátrico, nessa concepção, foi dividido, as comunidades esquadrinhadas, e a cada divisão hospitalar correspondia uma área geográfica e social da comunidade. Em cada área havia uma equipe de saúde mental e instituições extra hospitalares, para assegurar o tratamento (AMARANTE, 1995).
No entanto, a Psiquiatria Comunitária e Preventiva, a de Setor e a Comunidade Terapêtica, correntes de origem norte-americana, francesa e inglesa, respectivamente, "apesar de proporem uma nova racionalidade na assistência em saúde mental, não colocavam em xeque, sob qualquer hipótese, a essência mesma da psiquiatria enquanto saber e prática de intervenção. Não questionavam os conceitos mais fundamentais da psiquiatria 'oficial' mas, simplesmente, propunham uma modernização de suas formas de organização institucional" (AMARANTE, 1990: 78). Foram experiências reformadoras, mas que continuavam reiterando o modelo tradicional.

Os movimentos que representaram superação foram "a antipsiquiatria e as experiências surgidas a partir de Franco Basaglia*, enquanto instauradoras de rupturas com os movimentos anteriores, colocando em questão o próprio dispositivo médico-psiquiátrico e as instituições e dispositivos terapêuticos a ele relacionados" (AMARANTE, 1995: 29).

\section{Psiquiatria Democrática Italiana:}

Em 1962, Franco Basaglia e Antonio Slavich iniciaram em Gorizia - Itália, num hospital fechado de 700 leitos, um movimento de introdução dos principios da Comunidade Terapêutica. Foi um movimento eminentemente político, que criticava a organização capitalista da sociedade.

Esse movimento teve início, inaugurando na Itália uma experiência inovadora, a partir da autocritica ao insucesso da Comunidade Terapêutica aí implantada. Alguns aspectos marcaram sua diferença com os projetos de reforma, dentre eles, o da desinstitucionalização e o da negação/superação/ invenção da instituição (AMARANTE, s.d.).

Depois disso verificou-se uma rede de experiências inovadoras na Itália (Peruggia, Trieste, Arezzo, Reggio Emilia e Nápoles), constituindo-se assim a Psiquiatria Democrática Italiana. Tal movimento culminou com a Lei 180 de maio de 1978, proibindo a construção e utilização do manicômio como terapêutica, valorizando e reconhecendo a função essencial dos equipamentos extra hospitalares (PITTA-HOISEL, 1984).

Essa Lei estava incorporada à Reforma Sanitária Italiana, do mesmo ano de 1978, que norteou-se pelos princípios de: tutela de saúde de maneira unitária e global, através da prevenção e serviços básicos; redistribuição dos recursos técnicos, financeiros e humanos, para equilibrar as condições de saúde de toda a população; participação do cidadão na gestão da Unidade

\footnotetext{
* Franco Basaglia era um psiquiatra italiano que, juntamente com outro psiquiatra italiano - Antonio Slavich, propôs na Itália a superação dos princípios da Comunidade Terapêutica. Esse movimento inovador ficou conhecido como Psiquiatria Democrática Italiana.
} 
Sanitária Local e no planejamento, controle e execução das ações de saúde. Com relação à saúde mental, o Sistema Sanitário Nacional colocou como objetivo a sua tutela, privilegiando a prevenção e inserindo os serviços psiquiátricos nos serviços sanitários gerais (GOMES; SILVA FILHO, 1991).

Entendendo-se que a psiquiatria é composta por um conjunto de práticas que vão do organicismo à psiquiatria democrática e que ao adotar-se um novo modelo de atenção, este não se dá em detrimento do anterior, eles coexistem. Podemos identificar mais de uma corrente influenciando as ações interventivas dos profissionais, numa mesma instituição.

O que determina a escolha de uma corrente ou outra é a visão de mundo* dos profissionais e essa escolha determina o que esses profissionais tomarão como objeto de intervenção; quais serão os instrumentos utilizados nessa intervenção e que finalidade terá a assistência aos usuários dos serviços de saúde mental, nessa instituição.

As transformações ocorridas no processo de cuidar da enfermagem vem acompanhando as transformações ocorridas na prática do médico. Logo, o que vem sendo tomado como objeto dessa intervenção também não é o mesmo, olhando-se ao longo do tempo.

Tradicionalmente, a partir da segunda metade do século XIX, as práticas de enfermagem tem avançado "de acordo com as mudanças que acontecem na sociedade e nas ciências médicas" (BARROS, 1996:52). É dessa época, na Inglaterra, o início da história da enfermagem profissional, com Florence Nightingale**, que criou a primeira escola para formar enfermeiras, em 1860.

Antes disso as ações de enfermagem não estavam voltadas ao corpo enfermo, mas sim ao conforto da alma, tanto do doente quanto dos agentes de enfermagem (ALMEIDA; ROCHA, 1986). Foi a institucionalização do hospital como lugar de cura, que determinou a necessidade de pessoas mais qualificadas para auxiliar as ações do médico.

A institucionalização da enfermagem surgiu com a finalidade principal de disciplinar a conduta do pessoal que trabalhava nos hospitais, bem como o espaço do doente, com ventilação, água e higiene. A disciplina, que é um mecanismo de controle, era resultante da vigilância constante, espaço individualizador e classificativo e registro contínuo (ALMEIDA, ROCHA, 1986; MIRANDA, 1994); a disciplina passou a ser uma das características fundamentais desta profissão.
$\mathrm{Na}$ psiquiatria, quando os cuidados passaram a ser destinados ao corpo, criou-se a necessidade de pessoas habilitadas para aplicarem tais cuidados. A enfermagem psiquiátrica começou então a executar, além das ações disciplinadoras, os procedimentos específicos, desde aplicação de insulina, até a assistência às psicocirurgias, entre outros.

Ampliando as possibilidades terapêuticas dessas intervenções, centradas em procedimentos realizados no corpo do indivíduo acometido por um agravo mental, na década de 50 Hildegard Peplau, enfermeira americana, introduziu o relacionamento terapêutico enfermeira-paciente como o principal instrumento da enfermagem psiquiátrica. Esse instrumento foi desenvolvido a partir do entendimento da loucura proposto pela psicanálise e pela psiquiatria psicoterápica. O pressuposto do relacionamento terapêutico era o respeito mútuo entre enfermeira e clientes e o processo só era considerado efetivo se ambos saíssem acrescidos dessa relação (PEPLAU, 1988).

Este foi o primeiro modelo teórico sistematizado para a enfermagem psiquiátrica, desenvolvido por uma enfermeira. Este conhecimento exerceu grande influência no ensino de enfermagem psiquiátrica no Brasil.

A primeira escola de enfermagem no Brasil foi criada em 1890 no Rio de Janeiro, a Escola Profissional de Enfermeiros, no Hospício Nacional de Alienados. Assim, a primeira tentativa de sistematização do ensino da enfermagem brasileira foi na área psiquiátrica. O "locus" do trabalho era o hospício e os instrumentos da assistência de enfermagem eram o controle e a disciplina do doente mental. "Ficou assim caracterizada a função intermediária do enfermeiro entre os dois extremos da estrutura hospitalar - o médico e o paciente -, cumprindo as ordens que o primeiro prescrevia para o segundo" (BARROS, 1996: 60).

O processo fundante do ensino de enfermagem psiquiátrica deu-se nos moldes asilares, pautado na disciplina, na vigilância. Mesmo com a influência exercida pelo desenvolvimento da psicanálise nos programas de ensino, a partir da segunda metade da década de 50 , os campos de estágio permaneceram sendo os hospitais, com as atividades acadêmicas voltadas aos cuidados individuais, aos aspectos biológicos da doença. Mas, pode-se afirmar que as enfermeiras e a enfermagem, enquanto prestadoras da assistência psiquiátrica, têm sofrido influência das transformações ocorridas no campo dos saberes que embasam as práticas em psiquiatria (BARROS, 1996).

* Visão de mundo: "conjunto de princípios, pontos de vista e convicções que determina a atitude do homem em relação à realidade e a si próprio, a orientação da atividade de cada pessoa concreta, grupo social, classe ou sociedade em geral" (Krapivine apud FONSECA, 1990).

** Florence Nightingale, proveniente da burguesia inglesa, tinha conhecimentos - de linguas, de matemática, de contabilidade incomuns às mulheres de sua época. No contexto da medicina científica, ela propôs uma prática em saúde pautada num saber de enfermagem; sistematização do ensino e investigação empírica, cujos princípios nortearam a enfermagem moderna. 
No entanto, a formação acadêmica desenvolvida pelo sistema escolar mostra uma clara resistência a transformações. Entre a teoria adotada pelo órgão formador e o modelo de produção de serviços há uma contradição, os conhecimentos que subsidiam as ações práticas dos enfermeiros não correspondem, na sua totalidade, ao saber que vem sendo reproduzido pelo órgão formador (BARROS, 1996).

A mesma resistência pode ser detectada na formação de outros profissionais da saúde, uma vez que a disciplina de psiquiatria e análogas são fundamentadas em "doutrinas de racionalidade clinica, com sua redução ao âmbito exclusivamente biológico dos fenômenos relativos ao processo saúde / enfermidade mental", inviabilizando outras correntes que questionem esse paradigma (AMARANTE, 1990: 78).

A OPAS/OMS preconizou, em documento referente à reestruturação da atenção em saúde mental na América Latina, a necessidade de modificação do currículo, bem como a utilização de outros recursos assistenciais da comunidade, fora dos hospitais psiquiátricos, no treinamento e habilitação dos profissionais de saúde, para que tenha êxito a implantação de tal reestruturação (OPAS/OMS, s.d.). No Brasil o modelo, ainda hegemônico, utilizado na assistência aos portadores de transtornos psíquicos severos, é o clínico-biológico, acrescido de instrumentos inovadores, como o atendimento familiar, as atividades terapêuticas em geral, com entendimento pautado na psicanálise, mas ainda com a finalidade de remitir sintomas; logo, centrado na doença. Esse modelo tem sua legitimação no decreto de lei n. 24.559, de 3 de julho de 1934, "que foi promulgada num momento particularmente delicado no cenário politico-ideológico mundial e cuja finalidade é o seqüestro do sujeito do meio social e a sua exclusão dos centros urbanos" (SILVA, 1997: 4). Essa prática produziu uma cultura manicomial marginalizadora, excludente, que vem sendo reassegurada pelas instituições formadoras de profissionais de saúde e permeia o imaginário não só dos trabalhadores de saúde, como também dos próprios usuários, de seus familiares e da sociedade civil em geral.

O movimento que tem proposto no país ações que passam pela revisão das políticas públicas de saúde mental e dos modelos de assistência é a Reforma Psiquiátrica, que caracterizou-se - nos anos 80 - como o ápice de um processo de questionamentos, relativos ao caráter iatrogênico e cronificador e de exclusão social, do hospital psiquiátrico.

A Reforma Psiquiátrica para DELGADO (1992) e MACEDO (1997), contemplou vários aspectos, não se restringindo à formulação de políticas de saúde mental e sua tradução na transformação dos dispositivos assistenciais. Nessa direção, as propostas de Reforma Psiquiátrica estavam afinadas com um novo olhar sobre as partes constitutivas do processo de trabalho dos profissionais de saúde; qual seja, que fosse ampliado o objeto de trabalho para além da dimensão biológica da doença mental, com a finalidade de resgatar a singularidade dos indivíduos.

Pode-se afirmar, então, que a Reforma Psiquiátrica atualizou a dimensão de individualidade, tanto na sua dimensão jurídico política, cuja expressão máxima é a noção de cidadão, quanto à idéia de produção e cultivo da singularidade (MACEDO, 1997).

\section{2- Perspectivas para as ações em enfermagem}

Apesar de estar ainda aprendendo e desenvolvendo suas ações prioritariamente no modelo hegemônico, há um movimento, tanto dentro do hospital (humanização da assistência), quanto fora dele, no qual a enfermagem vem também desenvolvendo outras práticas, revendo conceitos.

Assim, este é o momento de rever o objeto de trabalho (constituir o sujeito enquanto cidadão), a prática (utilizar-se de novos instrumentos e com isso ampliar sua possibilidade de intervenção), e ampliar a finalidade da assistência, para além da remissão dos sintomas manifestos (CAMPOS, 1998).

A proposta de um novo paradigma - a Reforma Psiquiátrica - determina o repensar a prática e o saber que a embasa.

Com este entendimento, a enfermagem em saúde mental compartilha o objeto da assistência com a equipe multidisciplinar, cujo projeto é coletivo e determinado pela articulação das diferentes práticas (SILVA; FONSECA, 1995).

Assim, a enfermagem, "ao compor parcela do trabalho coletivo de saúde e seus integrantes parcela do trabalhador coletivo de saúde, não tem um processo próprio de trabalho, tampouco um objeto que lhe é especifico. Como as outras práticas sociais, ela tem responsabilidade de desenvolver sua especificidade nos processos de trabalho (assistência, gerenciamento, investigação e ensino) do processo de produção, processos de trabalho estes aos quais as demais práticas sociais também se conectam com suas especificidades" (QUEIROZ; SALUM, 1996).

Desta forma, os instrumentos de intervenção utilizados pelas enfermeiras há décadas, como o relacionamento interpessoal e a comunicação terapêutica, podem ser utilizados com eficácia, se inseridos no projeto terapêutico construido coletivamente pela equipe e usuário do serviço afinado com as necessidades deste, com a finalidade de que esse usuário possa acessar o direito à cidadania, considerando que não será um cidadão como qualquer outro. 
A prática da enfermeira, neste contexto, deve ser criativa, flexível, com finalidade de possibilitar aumento de habilidades, de autonomia do usuário do serviço de atenção à saúde mental, não mais voltada exclusivamente à remissão de sintomas.

É a escola que ensina a prática de enfermagem, ou seja, a assistência de enfermagem; o ensino é caracterizado como o paradigma do saber.

Considerando que o ensino retrata a visão de mundo dos docentes, visão esta que subjaz à concepção e à prática da assistência, torna-se fundamental a consciência do caráter ideológico do saber, tanto por quem ensina como por quem assiste (BARROS, 1996).

Por isso as(os) enfermeiras(os) precisam rever sua formação, apontando para novas posturas frente ao sujeito e seu sofrimento psíquico; ampliar conhecimentos para além dos adquiridos na graduação, compartilhando efetivamente práticas e saberes subjacentes à essas práticas, com outros campos disciplinares.

\section{REFERÊNCIAS BIBLIOGRÁFICAS}

ALMEIDA, M. C. P. de; ROCHA, J. S. Y. O saber de enfermagem e sua dimensão prática. São Paulo, Cortez, 1986.

AMARANTE, P. A (ainda) atualidade da psiquiatria democrática italiana. Saúde Deb.,n. 29, p. 77-80, 1990.

AMARANTE, P. D. de C. Novos tempos em saúde mental. Saúde Deb. n.37, p. 4, 1992.

AMARANTE, P. (org.) Loucos pela vida: a trajetória da reforma psiquiátrica no Brasil. Rio de Janeiro, PANORAMA/ENSP, 1995.

AMARANTE, P. D. de C. Análise dos determinantes e estratégias das politicas de saúde mental: o projeto da reforma psiquiátrica (1970-1990). Rio de Janeiro: Fundação Oswaldo Cruz, s.d. (Relatório parcial e alterações ao projeto de pesquisa). /mimeografado/.

BANDEIRA, M. Desinstitucionalização ou transinstitucionalização: lições de alguns países. J Bras Psiq., v. 40, n.7, p. 355-60, 1991.

BARROS, S. O louco, a loucura e a alienação institucional: o ensino de enfermagem sub judice. São Paulo, 1996. 201 p. Tese (Doutorado) - Escola de Enfermagem, Universidade de São Paulo.

CAMPOS, C.M.S. Consolidando a reforma psiquiátrica no Brasil através dos hospitais dia: a emergência das contradições entre a intencionalidade e a operacionalidade. São Paulo, 1998. 153 p. Dissertação (Mestrado) - Escola de Enfermagem, Universidade de São Paulo.

CAPLAN, G. Principios de psiquiatria preventiva. Rio de Janeiro, Zahar, 1980.

DELGADO, P. G. G. Perspectivas da psiquiatria pós-asilar no brasil. In: TUNDIS, S. A.; COSTA, N. do R. (org.). cidadania e loucura: politicas de saúde mental no Brasil. 3. ed. Petrópolis, Vozes, 1992. Cap. 5, p. 171-202.

DONNANGELO, M.C.F.; PEREIRA, L. Saúde e sociedade. São Paulo, Duas Cidades, 1976.

FONSECA, R.M.G.S. da Mulher, reprodução biológica e classe social: a compreensão do nexo coesivo através do estudo dialético do perfil reprodutivo biológico de mulheres atendidas nas Unidades Básicas de Saúde. São Paulo, 1990. 319p. Tese (Doutorado) - Escola de Enfermagem, Universidade de São Paulo.

GOMES, M.P.C.; SILVA FILHO, J. F. da Planejamento e saúde mental: o caso da organização dos serviços de saúde na Itália. J Bras. Psiq., v. 40 n. 8, p. 399-402, 1991.

GONÇALVES, R. B. M. Práticas de saúde: processos de trabalho e necessidades. Faculdade de Medicina, Universidade de São Paulo, 1992. /Mimeografado/

JONES, M. A comunidade terapêutica. Rio de Janeiro, Vozes, 1972.

MACEDO, A. L. P. reforma Psiquiátrica: uma breve discussão sobre impasses e contradições. In: VENANCIO, A. T.; LEAL, E. M.; DELGADO, P. G. (org.) O campo da atenção psicossocial. Rio de Janeiro, Te Corá, 1997.

MIRANDA, C. L. O parentesco imaginário: história e representação social da loucura nas relações do espaço asilar. São Paulo, Cortez, 1994.

ORGANIZACION PANAMERICANA DE LA SALUD Oficina Sanitaria Panamericana, Oficina Regional dela ORGANIZACION MUNDIAL DE LA SALUD. A reestruturação da atenção psiquiátrica na América Latina: uma nova política para os serviços de saúde mental. Washington, s.d. /mimeografado/.

PEDUZZI, M.; PALMA, J. J. L. da A equipe de saúde. In: SCHRAIBER, L. B.; NEMES, M. I. B.; MENDESGONÇAlveS, R. B. (org.) Saúde do adulto: programas e ações na unidade básica. São Paulo, Hucitec, 1996. p.234-50.

PEPLAU, H. E. Interpersonal relations in nursing. In: BELCHER, J. R.; FISH, L. J. B. Hildegard E. Peplau. New York. Springer Publishing, New York, 1988.

PITTA-HOISEL, A. M. Sobre uma politica de saúde mental. São Paulo, 1984. 161 p. Dissertação (Mestrado) - Faculdade de Medicina, Departamento de Medicina Preventiva, Universidade de São Paulo.

QUEIROZ, V. M. de; SALUM, M. J. L. Globalização econômica e a apartação na saúde: reflexão crítica para o pensar/fazer na enfermagem. Trabalho apresentado no $48^{\circ}$ Congresso Brasileiro de Enfermagem. São Paulo, 1996.

ROCHA, R. M. Enfermagem psiquiátrica: que papel é este? Rio de Janeiro, Instituto Franco Basaglia/ Te Corá, 1994.

SCHRAIBER, L. B. O médico e seu trabalho: limites da liberdade. São Paulo, Hucitec, 1993.

SILVA, A. L. A e; FONSECA, R.M.G.S. da a questão da interdisciplinaridade. Rev. Bras. Enf, v.48, n.3, p. 212-7, 1995.

SILVA, A. L.A. e O projeto copiadora do CAPS: do trabalho de reproduzir coisas à reprodução de vida. São Paulo, 1997. 131 p. Dissertação (Mestrado) - Escola de Enfermagem, Universidade de São Paulo.

SPINK, M. J. P. Saúde: um campo transdisciplinar? Rev. Terap. Ocup. USP., v.3, n. (1/2), p. 17-23, 1992. 